\title{
MULTICOLOR STUDY OF V1009 PER, A CLOSE BINARY SYSTEM AT THE BEGINNING OF THE OVERCONTACT PHASE, AND OF CRTS J031642.2+332639, A NEW BINARY SYSTEM IN THE SAME FIELD
}

\author{
Raúl Michel ${ }^{1}$, Francesco Acerbi ${ }^{2}$, Carlo Barani ${ }^{2}$, and Massimiliano Martignoni ${ }^{2}$ \\ Received June 6 2018; accepted January 82019
}

\begin{abstract}
The first multicolor observations and light curve solutions of the eclipsing binary systems V1009 Per and CRTS J031642.2+332639 are presented. Using the 2005 version of the Wilson-Devinney code, both systems are found to be W UMa contact binaries. V1009 Per has a mass ratio of $q=0.362 \pm 0.002$ and a shallow fill out parameter of $f=11.8 \pm 0.6 \%$ while CRTS J031642.2+332639 has a mass ratio of $q=2.507 \pm 0.006$ and a fill out of $f=13.6 \pm 0.4 \%$. High orbital inclinations, $i=85^{\circ} .9$ for V1009 Per and $i=83^{\circ} .2$ for CRTS J031642.2+332639, imply that both systems are total eclipsing binaries and that the photometric parameters here obtained are reliable. Based on 16 times of minimum the orbital period variations of V1009 Per are discussed. The absolute dimensions of the systems are estimated and, from the $\log M-\log L$ diagram, it is found that both components of the systems follow the general pattern of the W subtype W Ursae Majoris systems.
\end{abstract}

\section{RESUMEN}

Se presentan las primeras observaciones multicolor y las curvas de luz de las binarias eclipsantes V1009 Per y CRTS J031642.2+332639. Con la versión 2005 del código Wilson-Devinney se encuentra que ambos sistemas son binarias en contacto del tipo W UMa. El cociente de masas de V1009 Per es $q=0.362 \pm 0.002$ y el factor de llenado es $f=11.8 \pm 0.6 \%$; CRTS J031642.2+332639 tiene los valores $q=2.507 \pm 0.006$ y $f=13.6 \pm 0.4 \%$. Las inclinaciones orbitales, $i=85^{\circ} .9$ para V1009 Per e $i=83^{\circ} .2$ para CRTS J031642.2+332639, implican que ambas son binarias totalmente eclipsantes y que los parámetros fotométricos obtenidos son confiables. Con base en 16 tiempos de mínimo, se discuten las variaciones del período orbital de V1009 Per. Se calculan las dimensiones absolutas de los sistemas y, con el diagrama $\log M-\log L$, se encuentra que ambos componentes de los sistemas siguen el patrón general del subtipo W de las W Ursae Majoris.

Key Words: binaries: eclipsing — stars: individual: CRTS J031642.2+332639 stars: individual: V1009 Per - techniques: photometric

\section{INTRODUCTION}

The creation of a stellar evolutionary scheme requires a knowledge of the fundamental parameters of stars in different stages of their evolution. Eclipsing binary systems, especially the W Ursae Majoris type, are among the most important sources of such information (Kjurkchieva et al. 2017).

W UMa systems have orbital periods, typically, between 0.2 days and 0.8 days and consist of two

\footnotetext{
${ }^{1}$ Instituto de Astronomía, Universidad Nacional Autónoma de México, México.

${ }^{2}$ Stazione Astronomica Betelgeuse, Magnago, Italy.
}

dwarf stars with spectral types ranging from A to $\mathrm{K}$ sharing a common convective envelope resulting in a near equalization of the surface temperature with differences of no more than a few percent (Christopoulou et al. 2011).

Light curves of W UMa stars show continuous changes in brightness with nearly equal depth minima and maxima that are not always symmetric. This difference in maximum light levels, sometimes referred to as the O'Connell effect (O'Connell 1951), is caused by the inhomogeneity in the surface brightness distribution of one or both stars, commonly as- 
sociated with dark or hot spots. The difference between the maxima can change from orbit to orbit because of the motion and evolution of these active regions. This phenomenon may indicate the presence of an activity cycle similar to that of the Sun (Mitnyan et al. 2018).

According to our modern understanding, these systems are most likely formed from the moderately close binaries (Chen et al. 2016) through either nuclear evolution of the most massive component in the detached phase or angular momentum evolution of the two component stars within a convective envelope (Hilditch et al. 1988; Tutukov et al. 2004; Yildiz \& Doğan 2013).

V1009 Per (GSC 2344-00092, NSVS 6662264, $\left.\alpha_{2000}=03^{\mathrm{h}} 16^{\mathrm{m}} 49^{\mathrm{s}} .62, \delta_{2000}=+33^{\circ} 30^{\prime} 14^{\prime \prime} .1\right)$ was first reported by Kuruslov (2011) as a short-period contact eclipsing binary candidate with an orbital period of about 0.23414 days. The published light curve presented the typical EW-type behavior. However, the data of its light curve are scattered to some extent, and it is not clear that the light curve shows the O'Connell effect or argues about the features of the light curve at the time of the observations. We found the O'Connell effect in our observed light curves and adopted a bright spot model to interpret it.

CRTS J031642.2+332639 (hereinafter J031642, $\left.\alpha_{2000}=03^{\mathrm{h}} 16^{\mathrm{m}} 42^{\mathrm{s}} \cdot 23, \delta_{2000}=+33^{\circ} 26^{\prime} 39^{\prime \prime} .0\right)$ is listed as a variable star with a period of 0.3009380 days, in the Catalina Surveys Periodic Variable Star Catalog (Drake et al. 2014). This object also shows the typical behavior of W UMa type systems.

Since no photometric or spectroscopic studies are found in the literature, the aim of the present study is to analyze our $B, V, R_{c}$ and $I_{c}$ light curves to obtain the first orbital and fundamental parameters of these eclipsing binaries.

\section{OBSERVATIONS}

Photometric observations were carried out at the San Pedro Martir Observatory, on December 16, 2017 and January 18, 2018, with the 0.84-m telescope, a filter-wheel and the Spectral Instruments 1 CCD detector (a deep depletion e2v CCD42-40 chip with a gain of $1.39 \mathrm{e}^{-} / \mathrm{ADU}$ and a readout noise of $3.54 \mathrm{e}^{-}$). The field of view was $7.6^{\prime} \times 7.6^{\prime}$ and a binning of $2 \times 2$ was used during all the observations. Alternated exposures were taken in filters $B, V, R_{c}$ and $I_{c}$ with exposure times of 40,25, 15 and 15 seconds respectively. A total of 371 target images were acquired during the first night covering an interval of 5.7 hours while 414 images were acquired during

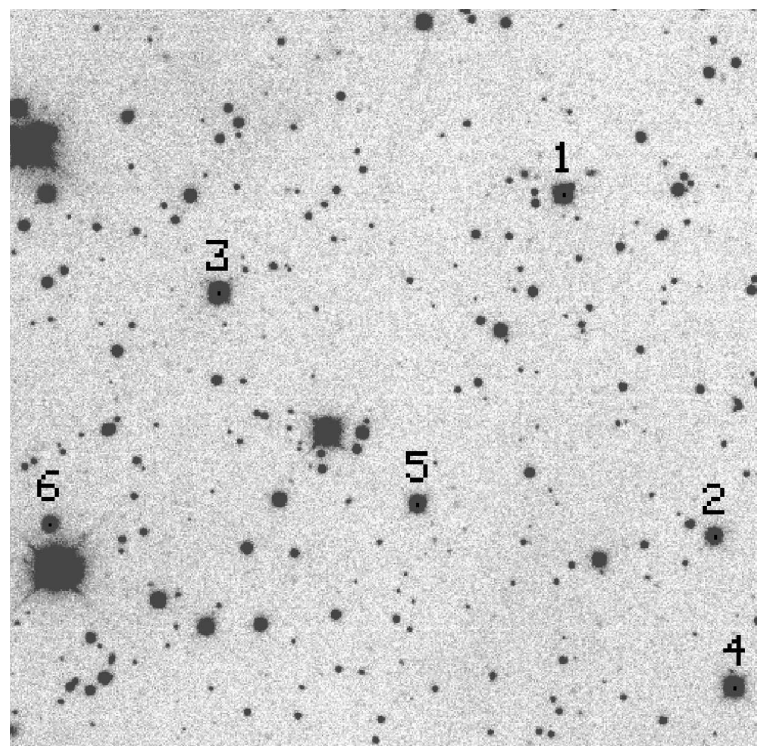

Fig. 1. Observed field. This finding chart was generated by aligning and adding all the images acquired during the second night of observation. The calibrated $U B V(R I)_{c}$ magnitudes of the marked stars can be found in Table 1 .

the second night over 6.3 hours. Flat field and bias frames were taken during both observing runs.

All images were processed using IRAF ${ }^{3}$ routines. Images were bias subtracted and flat field corrected before the instrumental magnitudes of the marked stars in Figure 1 were computed with the standard aperture photometry method. This field was also calibrated in the $U B V(R I)_{c}$ system and the results, along with the 2MASS magnitudes, are presented in Table 1. Based on this information, we decided to use Object \#3 as comparison star since it has a magnitude and color similar to both V1009 Per and J031642, making differential extinction corrections negligible. Objects $\# 4, \# 5$ and $\# 6$ were used as check stars to confirm that the comparison star is not variable. All data can be provided by the first author upon request.

\section{LIGHT ELEMENTS AND ORBITAL PERIOD VARIATIONS OF V1009 PER}

The measured times of minimum (ToM) of J031642, determined by the polynomial fits, are presented in Table 2. These new data permit us to refine the orbital period as:

$$
H J D(M i n . I)=2458136.8210(5)+0^{d} .2996181(83) \times E,
$$

\footnotetext{
${ }^{3}$ IRAF is distributed by the National Optical Observatories, operated by the Association of Universities for Research
} 
TABLE 1

$U B V(R I)_{C}$ AND 2MASS MAGNITUDES OF THE FIELD STARS. IDS AS IN FIGURE 1

\begin{tabular}{llcccccccccc}
\hline ID & Name & RA (2000) & DEC $(2000)$ & $U$ & $B$ & $V$ & $R_{c}$ & $I_{c}$ & $J$ & $H$ & $K_{s}$ \\
\hline 1 & V1009 Per & 49.206375 & 33.504269 & 15.938 & 15.406 & 14.351 & 13.716 & 13.112 & 12.307 & 11.734 & 11.619 \\
2 & CRTS J031642.2+332639 & 49.175527 & 33.444546 & 16.397 & 16.002 & 15.046 & 14.437 & 13.920 & 13.207 & 12.737 & 12.599 \\
3 & LAM260516231 & 49.278550 & 33.487820 & 15.482 & 15.071 & 14.109 & 13.567 & 13.042 & 12.228 & 11.779 & 11.681 \\
4 & LAM260516226 & 49.171755 & 33.418138 & 15.461 & 15.157 & 14.227 & 13.698 & 13.205 & 12.452 & 12.061 & 11.935 \\
5 & 2MASSJ03165701+3327025 & 49.237512 & 33.450702 & 16.434 & 15.955 & 14.987 & 14.458 & 13.988 & 13.186 & 12.837 & 12.680 \\
6 & 2MASSJ03171542+3326522 & 49.314234 & 33.447899 & 16.961 & 16.525 & 15.523 & 14.927 & 14.401 & 13.602 & 13.131 & 13.012 \\
\hline
\end{tabular}

TABLE 2

CCD (BVRI) TIMES OF MINIMA OF J031642

\begin{tabular}{ccc}
\hline HJD & Epoch & O-C \\
\hline 2458102.665 & -114 & 0 \\
2458136.671 & -0.5 & -0.0005 \\
2458136.822 & 0 & 0.0005 \\
\hline
\end{tabular}

TABLE 3

CCD (BVRI) TIMES OF MINIMA OF V1009 PER

\begin{tabular}{lcccl}
\hline Band & HJD & Epoch & O-C & Source \\
\hline Rotse & 2451491.536 & 0 & -0.0143 & Kuruslov (2011) \\
Rotse & 2451491.653 & 0.5 & -0.014 & NSVS \\
SWASP & 2453250.281 & 7511.5 & 0.0111 & SWASP \\
SWASP & 2453250.397 & 7512 & 0.0109 & SWASP \\
SWASP & 2454045.87 & 10909.5 & 0.0031 & SWASP \\
SWASP & 2454045.988 & 10910 & 0.0045 & SWASP \\
SWASP & 2454320.981 & 12084.5 & 0.0039 & SWASP \\
SWASP & 2454321.098 & 12085 & 0.0037 & SWASP \\
CCD & 2455846.852 & 18601.5 & 0.0043 & Diethelm (2012) \\
CCD & 2455846.968 & 18602 & 0.0031 & Diethelm (2012) \\
CCD(V) & 2456227.909 & 20229 & 0.0034 & Diethelm (2013) \\
CCD & 2457387.23 & 25180.5 & -0.0037 & Nosal P. \\
BVRI & 2458102.635 & 28236 & -0.0038 & This paper \\
BVRI & 2458102.752 & 28236.5 & -0.0046 & This paper \\
BVRI & 2458136.702 & 28381.5 & -0.0039 & This paper \\
BVRI & 2458136.819 & 28382 & -0.0037 & This paper \\
\hline
\end{tabular}

Based on a careful search for all available eclipsing times of V1009 Per, we collected a total of 16 ToM that, with the new four ToM observed by us (Table 3), permit the revision of the ephemeris and the construction of the O-C diagram depicted in Figure 2.

$H J D(M i n . I)=2451491.5503(37)+0^{d} .2341369(2) \times E$.

Applying the ephemeris of equation 2 to those minima, we notice that the trend of the residual has a parabolic shape as shown in Figure 2. A second order polynomial ephemeris, fitting all the minima, gives:

in Astronomy, Inc., under cooperative agreement with the National Science Foundation.

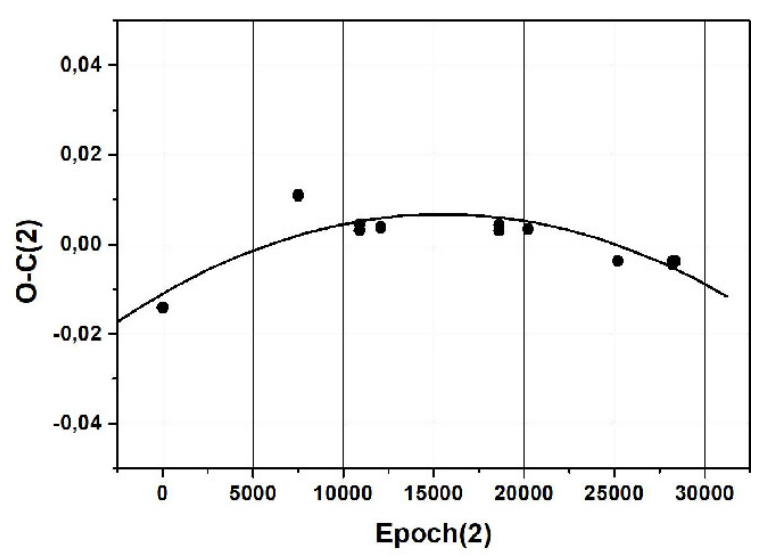

Fig. 2. O-C diagram of V1009 Per. The solid curve shows the second-order polynomial fit to the data points as given by equation 2 .

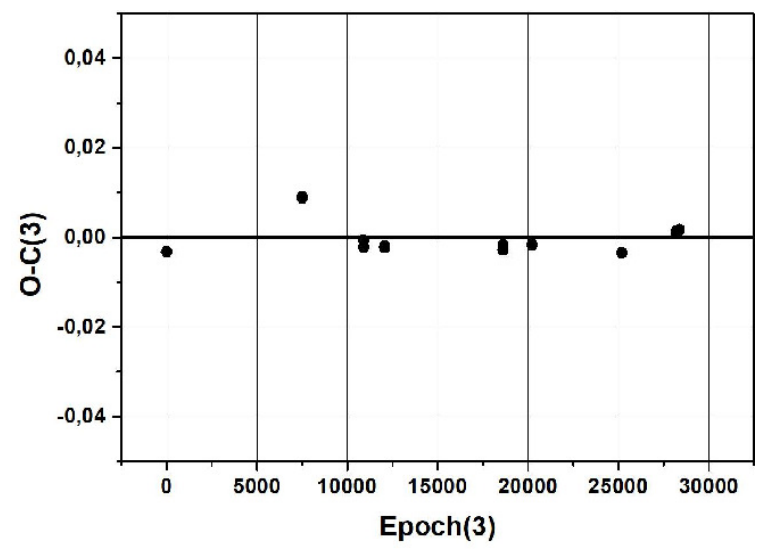

Fig. 3. O-C diagram of V1009 Per. The solid curve shows the second-order polynomial fit to the data points as given by equation 3 .

$$
\begin{aligned}
H J D(M i n . I)= & 2451491.5393(28)+0^{d} .2341392(4) \times \\
& E-7.4(1.2) \times 10^{-11} \times E^{2},
\end{aligned}
$$

whose residual behavior is shown in Figure 3. 

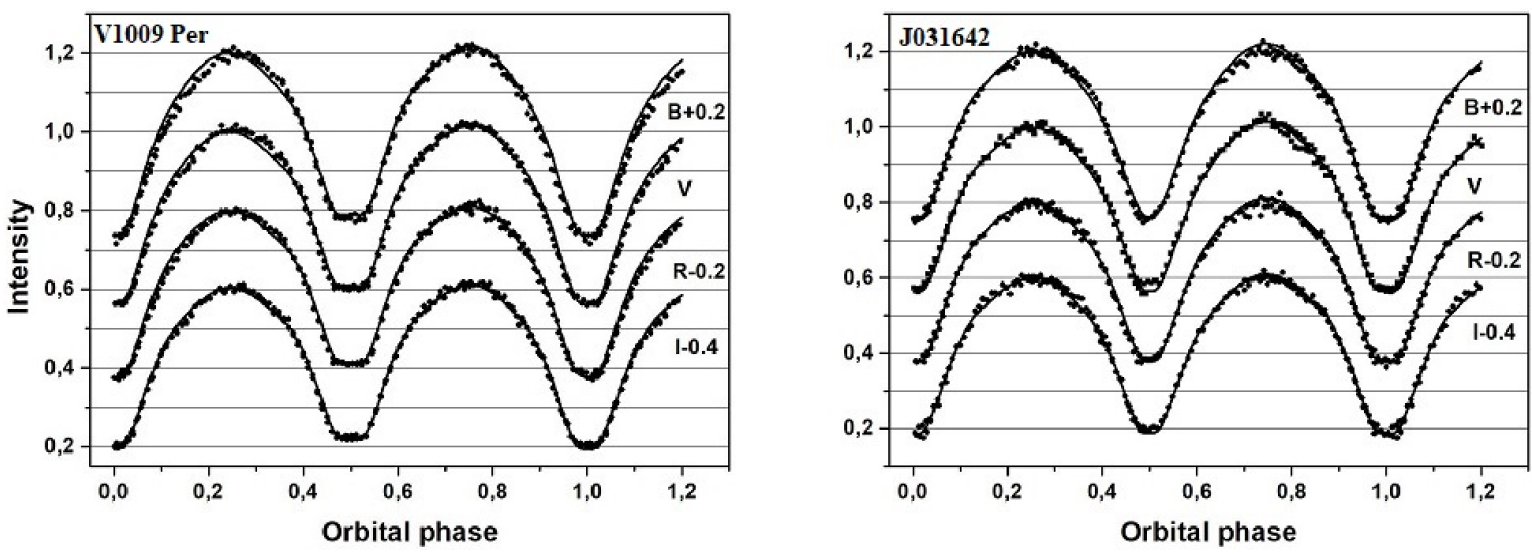

Fig. 4. CCD $B, V, R$ and $I$ light curves of V1009 Per (left) and J031642 (right). Points are the original observations and lines the theoretical light curves with the spot contribution.

Since the temporal distribution of the O-C values is rather small (covering only 18 years, spanning nearly 28,400 orbits), the downward parabolic change shown in Figure 2 may be only a part of a long-period cyclic oscillation that may be caused be the presence of a third body. To confirm this conclusion more times of light minimum are required.

From the quadratic term of equation (3), it follows that the orbital period may be decreasing at a rate of $\dot{P}=-2.31 \times 10^{-7}$ days $/ \mathrm{yr}^{-1}$. With the orbital period decrease, the primary component transfers mass to the secondary, the mass ratio increases and eventually the system evolves into the contact phase. The period decrease might be caused by mass or angular momentum loss (AML) due to a magnetic stellar wind (magnetic braking) and/or mass transfer from the more massive to the less massive component.

According to the formula given by Bradstreet \& Guinan (1994): $\dot{P}_{A M L}=-6.30 \times 10^{-9}$ days $/ \mathrm{yr}^{-1}$; this suggests that magnetic braking is not the main cause of the period decrease. The mass transfer from $M_{1}$ to $M_{2}$ or the mass loss from the system can be evaluated using equations 4 and 5 of Hilditch (2001) for conservative and non-conservative mass loss, respectively. We obtain for V1009 Per $\dot{M}_{1}=-1.63 \times$ $10^{-7} M_{\odot} \mathrm{yr}^{-1}$ and $\dot{M}_{1}=-1.91 \times 10^{-7} M_{\odot} \mathrm{yr}^{-1}$ for conservative and non-conservative mass transfer, respectively.

The timescale of the conservative mass transfer (i.e. the dynamical timescale) can be estimated to be approximately $\tau \mathrm{dy}=5.35 \times 10^{6} \mathrm{yrs}$ and $4.59 \times 10^{6} \mathrm{yrs}$ for the non-conservative mass transfer.

On the other hand, the thermal timescale of the massive component can be estimated as $\tau_{\text {th }} \approx 3.0 \times 10^{7}\left(M / M_{\odot}\right)^{2}\left(R / R_{\odot}\right)^{-1}\left(L / L_{\odot}\right)^{-1} \approx$ $5.8 \times 10^{7}$ yrs (Hilditch 2001) which is longer than the conservative mass-transfer duration. This suggests that the primary component cannot stay in thermal equilibrium and the mass transfer in V1009 Per is unstable.

\section{PHOTOMETRIC ANALYSIS USING THE WD CODE}

There are no reported spectroscopic mass ratios for these systems. In order to derive reliable geometric and astrophysical elements, the present observations were analyzed simultaneously using the 2003 (October 2005 revision) version of the WilsonDevinney (WD) program (Wilson \& Devinney 1971; Wilson 1990; Wilson 1994; Wilson \& van Hamme 2004).

We applied the $q$-search method to find the best initial value to be used for $q$ during the light curve analysis.

From Figure 4 left, it is clearly seen that the light curves of V1009 Per present a flatter bottom secondary eclipse covering approximately 0.07 in phase; this possibly indicates a total eclipse configuration of the system.

We used the NASA IPAC database (NASA 2015), interstellar extinction and reddening calculator to compute the $E(B-V)$ value for both the systems which, due to the low galactic latitude $\left(-20^{\circ}\right)$, could be significant.

We have checked the consistency of our determined $(B-V)_{0}$ values using the period-color relation discovered by Eggen and revised by Wang (1994) as $(B-V)_{0}=0.062-1.310 \log P($ days $)$. The results are the following: 

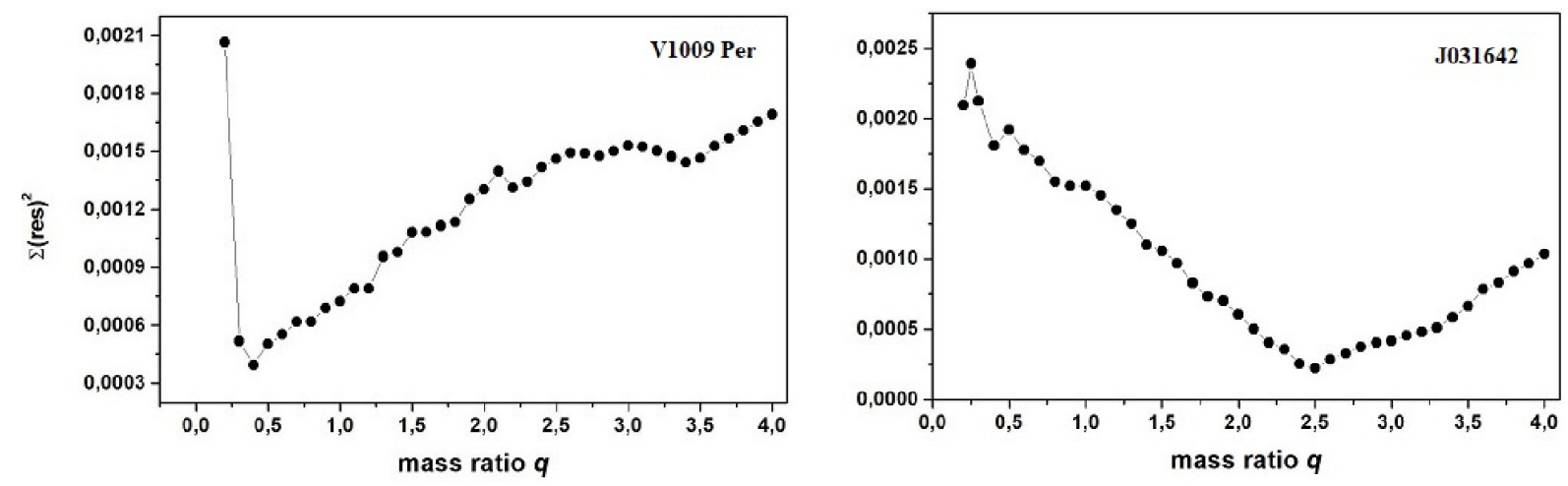

Fig. 5. The relation $\Sigma(\text { res })^{2}$ versus mass ratio $q$ in Mode 3 in the WD code for V1009 Per (left) and J031642 (right).

TABLE 4

\section{LIGHT CURVES SOLUTIONS FOR V1009 PER} AND J032642. ${ }^{1}$

\begin{tabular}{|c|c|c|}
\hline & V1009 Per & J032642 \\
\hline$i$ & $85.971 \pm 0.145$ & $83.245 \pm 0.148$ \\
\hline$T_{1}(K)$ & $5280^{*}$ & $5544^{*}$ \\
\hline$T_{2}(K)$ & $5253 \pm 6$ & $5416 \pm 6$ \\
\hline$\Omega_{1}=\Omega_{2}$ & $2.534 \pm 0.004$ & $5.872+0.009$ \\
\hline$q=m_{2} / m_{1}$ & $0.362 \pm 0.002$ & $0.398 \pm 0.006$ \\
\hline$A_{1}=A_{2}$ & $0.5^{*}$ & $0.5^{*}$ \\
\hline$g_{1}=g_{2}$ & $0.32^{*}$ & $0.32^{*}$ \\
\hline$L_{1 B}$ & $0.681 \pm 0.002$ & $0.318 \pm 0.002$ \\
\hline$L_{1 V}$ & $0.687 \pm 0.001$ & $0.311 \pm 0.001$ \\
\hline$L_{1 R}$ & $0.687 \pm 0.001$ & $0.308 \pm 0.001$ \\
\hline$L_{1 I}$ & $0.690 \pm 0.001$ & $0.307 \pm 0.001$ \\
\hline$L_{2 B}$ & $0.268 \pm 0.002$ & $0.634 \pm 0.003$ \\
\hline$L_{2 V}$ & $0.267 \pm 0.002$ & $0.639 \pm 0.003$ \\
\hline$L_{2 R}$ & $0.269 \pm 0.002$ & $0.645 \pm 0.002$ \\
\hline$L_{2 I}$ & $0.271 \pm 0.002$ & $0.654 \pm 0.002$ \\
\hline$f$ & $0.118 \pm 0.06$ & $0.136 \pm 0.04$ \\
\hline$X_{1 B}=X_{2 B}$ & $0.749^{*}$ & $0.624^{*}$ \\
\hline$X_{1 V}=X_{2 V}$ & $0.422^{*}$ & $0.319^{*}$ \\
\hline$X_{1 R}=X_{2 R}$ & $0.244^{*}$ & $0.168^{*}$ \\
\hline$X_{1 I}=X_{2 I}$ & $0.131^{*}$ & $0.075^{*}$ \\
\hline$L_{3}$ & 0 & 0 \\
\hline$r_{1}($ pole $)$ & $0.453 \pm 0.001$ & $0.289 \pm 0.001$ \\
\hline$r_{1}($ side $)$ & $0.488 \pm 0.001$ & $0.302 \pm 0.001$ \\
\hline$r_{1}(b a c k)$ & $0.591 \pm 0.001$ & $0.339 \pm 0.002$ \\
\hline$r_{2}($ pole $)$ & $0.289 \pm 0.001$ & $0.429 \pm 0.001$ \\
\hline$r_{2}($ side $)$ & $0.303 \pm 0.001$ & $0.470 \pm 0.001$ \\
\hline$r_{2}($ back $)$ & $0.247 \pm 0.003$ & $0.499 \pm 0.001$ \\
\hline lat spot $\left(^{\circ}\right)$ & $89 \pm 1.8$ & $51 \pm 2$ \\
\hline long $\operatorname{spot}\left({ }^{\circ}\right)$ & $310.1 \pm 3.1$ & $270.4 \pm 2.7$ \\
\hline $\operatorname{radius}\left({ }^{\circ}\right)$ & $24.8 \pm 0.92$ & $25.4 \pm 0.88$ \\
\hline Temp fac.Spot & $1.055 \pm 0.017$ & $1.03 \pm 0.02$ \\
\hline Star & 2 & 2 \\
\hline Sum (res)2 & 0.00030 & 0.00022 \\
\hline
\end{tabular}

${ }^{1}$ Assumed parameters are marked with *.
TABLE 5

\section{DIFFERENCES IN THE HEIGHT OF THE MAXIMA}

\begin{tabular}{lcc}
\hline & V1009 Per & J032642 \\
\hline Max II-Max I B & 0.008 & 0.011 \\
Max II-Max I V & 0.007 & 0.006 \\
Max II-Max I R & 0.016 & 0.008 \\
Max II-Max I I & 0.006 & 0.006 \\
\hline
\end{tabular}

For V1009 Per; NASA's calculator gives a value of $(B-V)_{0}=0.830$, i.e. $T_{1}=5280 K$, while Wang's equation gives $(B-V)_{0}=0.736$, i.e. $T_{1}=5140 \mathrm{~K}$ (a difference of $140 K$ ). For CRTS J031642; NASA's calculator gives $(B-V)_{0}=0.736$, i.e. $T_{1}=5544 K$, while Wang's equation gives $(B-V)_{0}=0.747$, i.e. $T_{1}=5505 K$ (a difference of $\left.39 K\right)$. The differences between the two values are smaller than the error bars. The $(B-V)$ color values obtained from our observations were corrected with the relative $E(B-$ $V)$ and the resulting values of $(B-V)_{0}$ were adopted for the determination of the temperature of Star 1.

Following Lucy (1967), the gravity-darkening coefficients of the two components were taken to be 0.32 and the bolometric albedo coefficients were set at 0.50 for stars with a convective envelope, $(\mathrm{Ru}-$ ciński 1973). Limb-darkening coefficients of the components were interpolated with a square root law from the van Hamme (1993) tables.

The shapes of the light curves of these systems are similar to the usual light curve shapes of the W UMa-type binary stars. This prompted us to start the W-D analysis directly in Mode 3. Mode 3 in the W-D Code is used for over-contact binaries 


\section{V1009 Per}
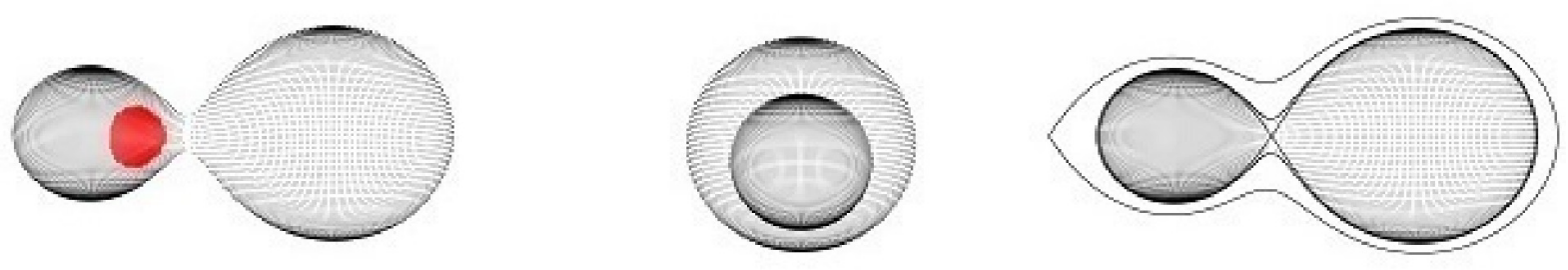

$\mathrm{J} 031642$
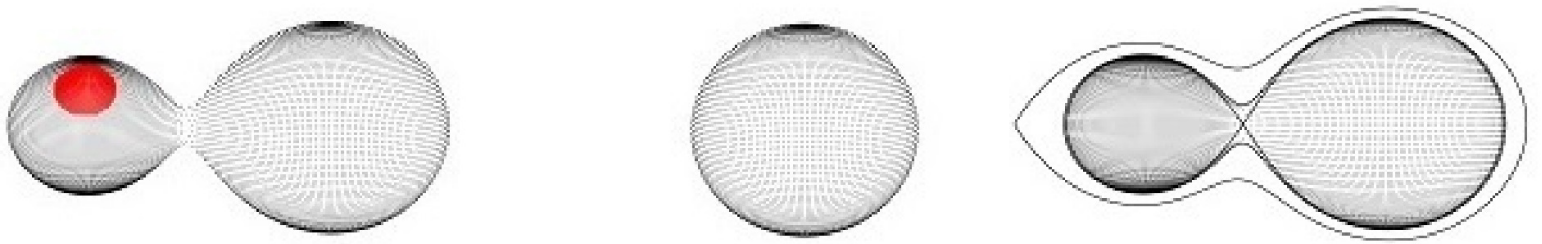

Fig. 6. Graphic representations of V1009 Per and J031642 according to our solution at quadrature (left) and at primary minimum (center). Right: the configuration of the components of the systems in the orbital plane is shown.

TABLE 6

ESTIMATED ABSOLUTE ELEMENTS FOR V1009 PER AND J031642

\begin{tabular}{lcccc}
\hline & \multicolumn{2}{c}{ V1009 Per } & \multicolumn{2}{c}{ J032642 } \\
\hline & Primary star & Secondary star & Primary star & Secondary star \\
Mass $\left(M_{\odot}\right)$ & $0.874 \pm 0.001$ & $0.317 \pm 0.007$ & $1.037 \pm 0.010$ & $0.414 \pm 0.030$ \\
Radius $\left(R_{\odot}\right)$ & $0.865 \pm 0.003$ & $0.474 \pm 0.003$ & $1.002 \pm 0.009$ & $0.661 \pm 0.014$ \\
Luminosity $\left(L_{\odot}\right)$ & $0.521 \pm 0.004$ & $0.153 \pm 0.002$ & $0.770 \pm 0.010$ & $0.370 \pm 0.020$ \\
$a\left(R_{\odot}\right)$ & \multicolumn{2}{c}{$1.694 \pm 0.006$} & \multicolumn{2}{c}{$2.133 \pm 0.029$} \\
\hline
\end{tabular}

(W UMa stars) in which the adjustable parameters used in the differential correction calculation are the orbital inclination, $i$, the mean surface effective temperature of the secondary component, $T_{2}$, the dimensionless surface potentials of the two components, $\Omega_{1}=\Omega_{2}$, and the monochromatic luminosity of the primary component $L_{1}$.

Due to the common occurrence of third bodies in WUMa systems (Pribulla \& Ruciński 2006), third light was included as an adjustable parameter. The results showed that the values for third light were negligible (smaller than the uncertainties). To search for a reliable mass ratio $q$, we made test solutions at the outset using the four light curves in $B V R_{c} I_{c}$ colors simultaneously.

The test solutions were computed for a series of assumed mass ratios $q$, with the values from 0.2 to 4 in steps of 0.1 for both systems; the behavior of the sum of squares of residuals, $\Sigma(r e s)^{2}$, was used to estimate their values. The relation between the resulting sum of weighted square deviations and $q$ is plotted in Figure 5. A minimum value was obtained at $q=0.40$ for V1009 Per and $q=2.5$ for J031642. Therefore, we chose the above initial values for the mass ratios $q$ and made them adjustable parameters. Then, we performed a differential correction until it converged and final solutions were derived (Table 4). It should be noted that the errors of the parameters given in this paper are the formal errors from the WD code and are known to be unrealistically small (Maceroni \& Ruciński 1997). For a discussion see Barani et al. (2017).

The results of our analysis confirm that both the system are shallow contact binaries in good thermal contact. For systems exhibiting high inclination, the mass ratios can be inferred from purely geometric arguments even in the absence of complementary spectroscopic data (Terrell \& Wilson 2005). As shown in Figure 4, the light curves of both systems display an inverse O'Connell effect (O'Connell 1951). The maximum at phase 0.25 (Max I) is slightly fainter than the one at phase 0.75 (Max II), see Table 5. 
These features usually indicate wavelengthdependent hot spot activity (rather than a cool spots) on the surface of one component due to the probable impact from the mass transfer between the components. The final synthetic light curves calculated using the whole set of parameters of Table 4 are shown in Figure 4 as continuous lines. The observed and the theoretical light curves are in good agreement. A graphic representations and the Roche geometries of the systems is shown in Figure 6.

\section{EVOLUTIONARY STATUS OF THE SYSTEMS AND CONCLUSIONS}

Since at present there is no spectroscopic determination of the orbital elements available, the absolute parameters of the system cannot be determined directly. We use the 3D empirical laws of Gazeas (2009) where the physical parameters of contact binaries are closely correlated with the orbital period and mass ratio. Then with the mass ratio determined from the photometry, we can derive the individual masses and radii.

Luminosities were calculated using the StefanBoltzmann law. The physical parameters listed in Table 6 are used to investigate the current evolutionary status of both systems.

Recently, Yildiz \& Doğan (2013), developed a method for the computation of initial masses of contact binaries based on stellar modelling with mass loss. Their main assumption was that the mass transfer starts near or after the TAMS phase of the massive component (the progenitor of the secondary component). They found that binary systems with initial mass of the secondary, $M_{2 i}$, higher than $1.8 M_{\odot}$ become A-subtype, but if $M_{2 i}$ is lower than $1.8 \mathrm{M}_{\odot}$ then the systems exhibit $\mathrm{W}$-subtype properties. Applying this method we find $M_{2 i}=1.3 M_{\odot}$ for V1009 Per and $M_{2 i}=1.4 M_{\odot}$ for J031642. These results agree with the determination of the $\mathrm{W}$-subtype based on the above criterion for the initial mass of the secondary.

In Figure 7 we plot the components of V1009 Per and J031642 together with other W-and A-type W UMa systems collected by Yildiz \& Doğan (2013) in the logarithmic mass-luminosity $(M-L)$ relations along with the ZAMS and TAMS computed by Girardi et al. (2000). It is clear that both components of our systems follow the general pattern of the Wsubtype systems and seem to be in good agreement with the well known W-type W UMa systems on the $\log M-\log L$ plane.

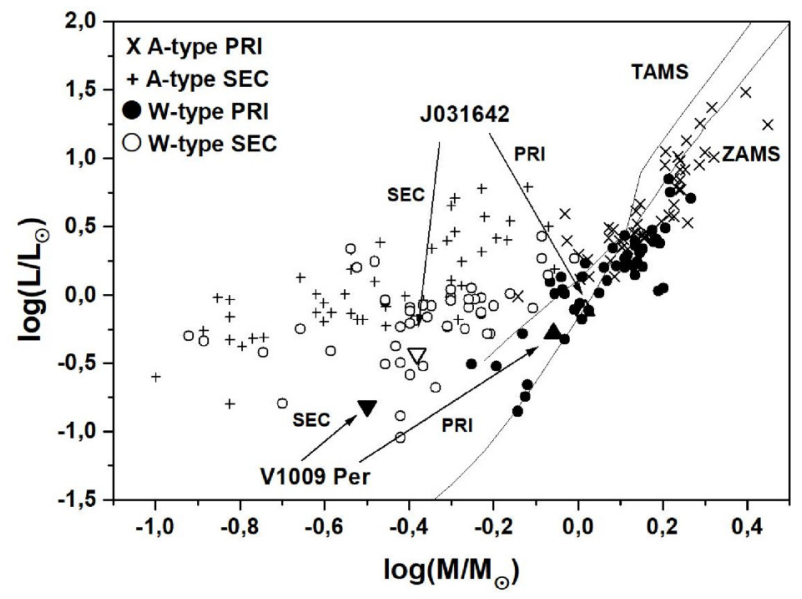

Fig. 7. Location of the components of V1009 Per and J031642 on the $\log M-\log L$ diagram. The sample of W UMa type systems was obtained from a compilation of Yildiz \& Doğan (2013) Zero Age Main Sequence (ZAMS) and Terminal Age Main Sequence (TAMS) are taken from Girardi et al. (2000) for the solar chemical composition.

The light curves of both systems exhibit the inverse O'Connell effect with the maximum at phase 0.25 (Max I) slightly fainter than that at phase 0.75 (Max II). For this reason a hot spot, indicating a probable impact from mass transfer between the components, was placed on the surface of the secondary component.

RM acknowledges financial support from UNAM under DGAPA grant PAPIIT IN 100918. This paper is based on observations acquired at the OANSPM, Baja California, Mexico. This research has made use of the International Variable Star Index (VSX) database, operated at AAVSO, Cambridge, Massachusetts, USA, of the VizieR catalogue access tool, CDS, Strasbourg, France. The original description of the VizieR service was published in A\&AS 143,23 . We acknowledge our anonymous referee for comments that helped to improve this work.

\section{REFERENCES}

Barani, C., Martignoni, M., \& Acerbi, F. 2017, NewA, 50, 73

Bradstreet, D. H. \& Guinan, E. F. 1994, ASPC 56, Interacting binaries stars, ed. A. W. Shafter (San Francisco, CA: ASP), 228

Chen, X., Deng, L., de Grijs, R., et al. 2016, AJ 152, 129 
Christopoulou, P.-E., Parageorgiou, A., \& Chrysopoulos, I. 2011, AJ 142, 99

Diethelm, R. 2012, IBVS, 6011, 1 2013, IBVS, 6042, 1

Drake, A. J., Graham, M. J., Djorgovski, S. G., et al. 2014. ApJS, 213, 9

Gazeas, K. D. 2009, CoAst, 159, 129

Girardi, L., Bressan, A., Bertelli, G., \& Chiosi, C. 2000, A\&AS 141, 371

Hilditch, R. W., King, D. J., \& McFarlane, T. M. 1988, MNRAS, 231, 341

Hilditch, R. W. 2001, An introduction to Close Binary Stars, (Cambridge, MA: CUP)

Kjurkchieva, D. P., Popov, V. A., Ibryamov, S. I., Vasileva, D. L., \& Petrov, N. I. 2017, RAA, 17, 42

Kuruslov, A. V. 2011, PZP, 11, 1

Lucy, L. B. 1967, ZA, 65, 89

Maceroni, C. \& Ruciński, S. M. 1997, PASP, 109, 782

Mitnyan, T., Bódi, A., Szalai, T., et al. 2018, A\&A, 612, 91 NASA. 2015, http://irsa.ipac.caltech.edu/
applications/DUST/

Nosal, P., O-C gateway, http://var2.astro.cz/ ocgate/

O'Connell, D. J. K. 1951, PRCO, 2, 85

Pribulla, T. \& Ruciński, S. M. 2006, AJ, 131, 2986

Ruciński, S. M. 1973, AcA, 23, 79

Terrell, D. \& Wilson, R. E. 2005, Ap\&SS, 296, 221

Tutukov, A. N., Dremova, G. N., \& Svechnikov, M. A. 2004, ARep, 48, 219

van Hamme, W. 1993, AJ, 106, 2096

Wang, J. M. 1994, ApJ, 434, 277

Wilson, R. E. \& Devinney, E. J. 1971, ApJ, 166, 605

Wilson, R. E. 1990, ApJ, 356, 613 1994, PASP, 106, 921

Wilson, R. E. \& van Hamme, W. 2004, Computing binary stars observables, http://ftp.astro.ufl.edu, directory pub/wilson/lcdc2003

Worthey, G. \& Lee, H. C. 2011, ApJs, 193, 1

Yildiz, M., \& Doğan, T. 2013, MNRAS, 430, 2029

F. Acerbi: Via Zoncada 51, Codogno, LO, 26845, Italy (acerbifr@tin.it).

C. Barani: Via Molinetto 35, Triulza di Codogno, LO, 26845, Italy (cvbarani@alice.it).

M. Martignoni: Via Don G. Minzoni 26/D, Magnago, MI, 20020, Italy (massimiliano.martignoni@alice.it).

R. Michel: Universidad Nacional Autónoma de México. Observatorio Astronómico Nacional. Apartado Postal 877, C.P. 22800, Ensenada, B. C., México (rmm@astro.unam.mx). 